\title{
A MODELAGEM MATEMÁTICA COMO PROPOSTA PEDAGÓGICA NA PERSPECTIVA DO ENSINO DA MATEMÁTICA EM AMBIENTES INFORMATIZADOS NO ENSINO MÉDIO
}

\author{
MATHEMATICAL MODELING AS A PEDAGOGICAL PROPOSAL IN THE PERSPECTIVE OF \\ TEACHING MATHEMATICS IN COMPUTERIZED ENVIRONMENTS IN HIGH SCHOOL
}

\section{EL MODELADO MATEMÁTICO COMO PROPUESTA PEDAGÓGICA DESDE LA PERSPECTIVA DE LA ENSEÑANZA DE LAS MATEMÁTICASEN AMBIENTES INFORMÁTICOS EN LA ESCUELA SECUNDARIA}

Rafaela Silva Bezerra do Nascimento ${ }^{1}$

Submetido em: $18 / 10 / 2021$

e211905

Aprovado em: 28/11/2021

https://doi.org/10.47820/recima21.v2i11.905

\section{RESUMO}

A presente pesquisa aborda e analisa as contribuições da modelagem matemática como proposta pedagógica na perspectiva do ensino da matemática em ambientes informatizados no Ensino Médio. Para argumentar acerca dos objetivos específicos que são: descrever as estratégias utilizadas pelos professores da Escola Fábio da Silveira Barros para ensinar matemática no ensino médio; elaborar uma proposta pedagógica com desenvolvimento de atividades de Modelagem Matemática em ambientes informatizados, respaldada na Educação Matemática; e descrever os resultados alcançados com a prática da nova metodologia apresentada. Esta deu-se através de uma pesquisa descritiva de corte transversal, onde é possível ao investigador, registrar e analisar as características dos mesmos indivíduos em diferentes momentos, proporcionando maior compreensão sobre o tema abordado. Realizada em forma de pesquisa ação, com enfoque misto, onde possibilitou um estudo de uma situação social com o objetivo de melhorar a qualidade da ação desenvolvida no seu interior. Os participantes da pesquisa foram 03(três professores) e 30 (trinta) estudantes. Na intenção de alcançar respostas acerca dos questionamentos abordados nessa pesquisa, foram utilizados vários instrumentos para a coleta de dados, como: aplicação do questionário inicial ao estudante, realizado antes da proposta pedagógica e o questionário final após a vivência das atividades; a observação participante, que transcorreu durante o desenvolvimento das atividade com modelagem matemática etecnologia, para promover a aproximação e a interação dos fatos da realidade com o conteúdo matemático; e a entrevista, que foi realizada com os professores. Os resultados dessa pesquisa mostraram contribuições significativas da Modelagem Matemática a partir de temas para a abordagem de conteúdo matemático, e contribuições das tecnologias para 0 desenvolvimento do ambiente de aprendizagem com Modelagem Matemática. Dessa forma, a pesquisa permitiu aos estudantes um novo olhar sobre a matemática, baseado em uma proposta de modelagem matemática com aportes tecnológicos, proporcionando a interação do conteúdo com a realidade, resultando em uma aprendizagem siginificativa.

PALAVRAS-CHAVE: Ensino Aprendizagem. Modelagem Matemática. Tecnologia

\begin{abstract}
This research approaches and analyzes the contributions of mathematical modeling as a pedagogical proposal from the perspective of mathematics teaching in computerized environments in high school. To argue about the specific objectives: describe the strategies used by teachers of the Fábio da Silveira Barros School to teach mathematics in high school; develop a pedagogical proposal with the development of mathematical modeling activities in computerized environments, supported by Mathematics Education; and describe the results achieved with the practice of the new methodology presented. This was through a descriptive cross-sectional research, where it is possible for the researcher to record and analyze the characteristics of the same individuals at different times, providing greater understanding of the theme addressed. Carried out in the form of action research, with a mixed focus, where it allowed a study of a social situation with the objective of improving the quality of the action developed inside it. The participants
\end{abstract}

\footnotetext{
1 Especialista no Ensino da Matemática - Universidade Federal Rural de Pernambuco - Brasil sb_rafinha@hotmail.com; investigação apresentada ao Programa de Pós Graduação de Maestria em Ciências da Educação, da Universidade Autônoma de Assunção.
}

RECIMA21 - Ciências Exatas e da Terra, Sociais, da Saúde, Humanas e Engenharia/Tecnologia 


\section{RECIMA21 - REVISTA CIENTÍFICA MULTIDISCIPLINAR ISSN 2675-6218}

A MODELAGEM MATEMÁTICA COMO PROPOSTA PEDAGÓGICANA PERSPECTIVA DO ENSINO DA MATEMÁTICA EM AMBIENTES INFORMATIZADOS NO ENSINO MÉDIO
Rafaela Silva Bezerra do Nascimento

of the research were 03 (three teachers) and 30 (thirty) students. In order to reach answers about the questions addressed in this research, several instruments were used for data collection, such as: application of the initial questionnaire to the student, performed before the pedagogical proposal and the final questionnaire after the experience of the activities; participant observation, which occurred during the development of activities with mathematical modeling and technology, to promote the approximation and interaction of the facts of reality with the mathematical content; and the interview, which was conducted with the teachers. The results of this research showed significant contributions of Mathematical Modeling from themes to the mathematical content approach, and contributions of technologies to the development of the learning environment with Mathematical Modeling. Thus, the research allowed students a new look at mathematics, based on a proposal of mathematical modeling with technological contributions, providing the interaction of content with the reality, resulting in a significative learning.

\section{KEYWORDS: Teaching Learning. Mathematical Modeling. Technology}

\section{RESUMEN}

Esta investigación aborda y analisa las contribuciones del modelado matemático como una propuesta pedagógica desde la perspectiva de la enseñanza de las matemáticas en entornos computarizados enla escuela secundaria. Discutir sobre los objetivos específicos que son: describir las estrategias utilizadas por los maestros en la Escuela Fábio da Silveira Barros para enseñar matemáticas en la escuela secundaria; elaborar una propuesta pedagógica con el desarrollo de actividades de Modelado Matemático en entornos computarizados, con el apoyo de la Educación Matemática; Los resultados logrados con la práctica de la nueva metodología presentada, esto fue a través de una investigación transversal descriptiva, donde es posible que el investigador registre y analice las características de los mismos individuos en diferentes momentos, proporcionando una mayor comprensión del tema abordado. Realizado en forma de investigación de acción, con enfoque mixto, donde permitió un estudiode una situación social con el objetivo de mejorar la calidad de la acción desarrollada dentro de ella. Los participantes de la investigación fueron 03 (tres profesores) y 30 (treinta) estudiantes. Para obtener respuestas sobre las preguntas abordadas en esta investigación, se utilizaron varios instrumentos parala recopilación de datos como aplicación del cuestionario inicial al estudiante, realizado antes de la propuesta pedagógica y el cuestionario final después de las actividades, la observación participante que tuvo lugar durante El desarrollo de actividades con modelos matemáticos y tecnología, para promover la aproximación e interacción de los hechos de la realidad con el contenido matemático y la entrevista que se realizó con los docentes. Los resultados de esta investigación mostraron contribuciones significativas del modelado matemático desde los temas hasta el enfoque del contenido matemático, y las contribuciones de las tecnologías al desarrollo del entorno de aprendizaje con el modelado matemático. Por lo tanto, la investigación permitió a los estudiantes una nueva mirada sobrelas matemáticas basada en una propuesta de modelado matemático con contribuciones tecnológicas, proporcionando la interacción del contenido con el realidad, lo que resulta en un aprendizaj e significativo.

PALABRAS CLAVE: Enseñanza aprendizaje. Modelación matemática. Tecnologia.

\section{INTRODUÇÃO}

É perceptível as dificuldades que o ensino da matemática vem enfrentando faces a um mundo de avanços tecnológicos, portadora de uma metodologia tradicional empregada com frequência pelos agentes que promovem o ensino, esta metodologia, por sua vez, é um dos principais entraves no processo de ensino aprendizagem, promovendo uma educação monológica, linear, fragmentada e mecanizada. Ainda existe uma distância enorme da matemática estudada na escola com a matemáticada vida real, os alunos não conseguem fazer relação do conteúdo estudado com seu cotidiano.

Diante deste cenário, essa pesquisa aborda questões específicas sobre a metodologia de ensino utilizada pelos professores do ensino médio durante a ministração de aulas da disciplina matemática da Erem Fábio da Silveira Barros, e a inserção de materiais tecnológicos como suporte pedagógico, tendo como temática A Modelagem Matemática como Proposta Pedagógica na perspectiva do Ensino da 


\section{RECIMA21 - REVISTA CIENTÍFICA MULTIDISCIPLINAR ISSN 2675-6218}

A MODELAGEM MATEMÁTICA COMO PROPOSTA PEDAGÓGICANA PERSPECTIVA DO ENSINO DA MATEMÁTICA EM AMBIENTES INFORMATIZADOS NO ENSINO MÉDIO

Matemática em Ambientes Informatizados no ensino médio.

O ensino da Matemática é visto como uma linguagem capaz de traduzir a realidade e estabelecer suas diferenças. A aplicação em contextos diferentes daqueles em que foram adquiridos exige muito mais que a simples decoração ou a solução mecânica de exercício, a exemplo de: domínio de conceitos, flexibilidade de raciocínio, capacidade de análise e abstração. Essas capacidades são necessárias em todas as áreas de estudo.

Existe uma preocupação de toda comunidade escolar com o cumprimento do conteúdo, que são muitos, não importa como aconteça, o importante é que de fato o conteúdo seja dado. No entanto percebese que o ensino da Matemática deve ir além de reprodução, memorização e treinamento dosexercícios contidos nos livros didáticos.

É importante salientar sobre a Matemática do Ensino Médio:

a relação desproporcional entre a quantidade de conteúdo programático e o tempo hábil para desenvolvê-lo fomenta uma prática docente para o ensino da Matemática, em que o professoresforça-se para transmitir ao aluno todo o conteúdo de forma pronta, acabada e acelerada, elevando, para o primeiro plano da ação docente, o conteúdo, e rebaixando para o segundo, a aprendizagem (CHAVES, 2005, p. 11).

Nesse contexto, cabe ao professor planejar situações com sentido, isto é, que tenham significado para os estudantes e escolher materiais que sirvam de apoio para o trabalho que eles realizarão nas aulas com esses conteúdos. Atividades que propiciem ao estudante, sua manifestação para os problemas que desencadeiam suas atividades intelectuais.

Consideremos que só é possível desencadear ideias matemáticas na cabeça de alguém, se esse alguém é colocado diante de uma situação envolvente que lhes seja provocadora, interessante, e ao mesmo tempo, que seja capaz de estimular a aprendizagem. Não é usar situações abstratas, como explicada oralmente, posta no livro didático, exposta na lousa. Tem que ser algo que desperte no estudante $o$ interesse pela busca de possíveis soluções, promovendo assim uma aprendizagem plena, espontânea.

De acordo com os PCNs temos que:

O fato de o aluno ser estimulado a estimular sua própria resposta, questionar o problema, a transformar um dado problema uma fonte de novos problemas, evidencia uma concepção de ensino e aprendizagem não pela mera reprodução de conhecimento mais pela via da ação refletida que constrói conhecimento (PCNs, 2001).

Nisso, a matemática apresentada em sala de aula só será entendida quando esta traz um significado para o aluno. Logo, o educador, enquanto articulador da construção desse conhecimento deve conhecer a realidade com a qual vai trabalhar, isso significa que inicialmente ele tem que aprender com seus alunos.

Na busca de resolver essa problemática em estudo, e de mostrar ao aluno e consequentemente a comunidade escolar em geral um significado mais amplo sobre o ensino da matemática e seu uso no cotidiano, algumas propostas atuais, sugerem o uso da Modelagem no ensino da matemática, pois, ela torna o aluno mais consciente da utilidade da matemática para resolver e analisar problemas do dia-adia.

De acordo com Franchi (1993, p.1): 


\section{RECIMA21 - REVISTA CIENTÍFICA MULTIDISCIPLINAR ISSN 2675-6218}

A MODELAGEM MATEMÁTICA COMO PROPOSTA PEDAGÓGICANA PERSPECTIVA DO ENSINO DA MATEMÁTICA EM AMBIENTES INFORMATIZADOS NO ENSINO MÉDIO

"o objetivo principal da educação escolar é habilitar o estudante para uma participação crítica eativa na sociedade em que vive. Assim, empreendi uma busca de perspectivas metodológicasdiferenciadas que contribuíssem com o desenvolvimento da capacidade de pensar e questionardos estudantes e que também dinamizem o ambiente das aulas de Matemática".

Diante disso, fomenta a busca por alternativas de trabalhar os conceitos matemáticos de modo que venha possibilitar uma aprendizagem significativa, ajudando a promover o progresso da sociedade. Assim, a Modelagem Matemática se apresenta como uma maneira de contextualizar os conceitos matemáticos, possibilitando assim interpretação e ação na realidade D'ambrósio, (1986). Por outro lado, as tecnologias de informação e comunicação configuravam-se como recursos interessantes para as atividades de Modelagem, possibilitando a exploração de conceitos matemáticos (FRANCHI, 2007; ARAÚJO, 2009; MALHEIROS, 2004). Essas abordagens nos remetem a estruturar uma proposta pedagógica para o ensino de Matemática baseada em ambos os conceitos.

Nisso, destacamos o seguinte:

Quando o professor aplica a modelagem como estratégia pedagógica na sala de aula, ele tema intenção de ensinar matemática. Ao explorar as aplicações matemáticas no dia-a-dia, a construção de modelos e o relacionamento entre a matemática utilizada na modelagem e o conteúdo programático, o professor oferece ao aluno a oportunidade de conviver com um conteúdo vivo, prático, útil e com bastante significado. (JACOBINI, 2004, p. 22).

Por conseguinte, é necessário elucidar caminhos para o desenvolvimento da prática do ensinoaprendizagem evidenciando o uso das tecnologias de informação e comunicação a fim de criar possibilidades para efetivação e significado a educação.

De acordo com Franchi (2007), é importante construir ambientes de aprendizagem com utilização de tecnologias que permitam a interação do aluno com o conhecimento, a participação no desenvolvimento das atividades, a exploração de informações à sua maneira. Nesse tipo de ambiente, alunos e professores aprendem juntos.

Assim, as ferramentas tecnológicas devem ser associadas à construção do conhecimento, preparando o cidadão do futuro para uma vida social e profissional, através de um ambiente que propicie a aprendizagem. Assim, faz-se necessário que haja uma evolução das instituições escolares para acompanhar o desenvolvimento social, onde as novas tecnologias estão cada vez mais presentes.

Nesse contexto, a elaboração e implementação da proposta pedagógica, bem como a análise dos resultados obtidos constituem, pois, objeto desta pesquisa. Assim, a questão que orienta a condução das atividades dessa investigação é:

Que contribuições uma proposta pedagógica baseada na Modelagem Matemática em ambientes informatizados pode trazer para a construção do conhecimento matemático de maneira significativa? Essa questão delineou a escolha dos procedimentos adotados, da metodologia de pesquisa a ser utilizada e dos instrumentos de coleta de dados.

\section{Objetivo Geral:}

Analisar a contribuição da utilização da Modelagem Matemática em ambientes informatizados na construção do conhecimento matemático de alunos de Ensino Médio na Escola Fábio da Silveira 


\section{RECIMA21 - REVISTA CIENTÍFICA MULTIDISCIPLINAR ISSN 2675-6218}

A MODELAGEM MATEMÁTICA COMO PROPOSTA PEDAGÓGICANA PERSPECTIVA DO ENSINO DA MATEMÁTICA EM AMBIENTES INFORMATIZADOS NO ENSINO MÉDIO

Barros, ano 2019.

Objetivos Específicos:

1. Descrever as estratégias utilizadas pelos professores da Escola Fábio da Silveira Barros para ensinar matemática no ensino médio;

2. Elaborar uma proposta pedagógica com desenvolvimento de atividades de Modelagem Matemática em ambientes informatizados, respaldada na Educação Matemática;

3. Descrever os resultados alcançados com a prática da nova metodologia apresentada.

\section{METODOLOGIA}

O desenho metodológico é não experimental, porque não se manipula variáveis. Descritiva, pois descreve os fatos e fenômenos sobre uma determinada realidade. De acordo com Gil (2008), as pesquisas descritivas possuem como objetivo a descrição das características de uma população, fenômeno ou de uma experiência. Ao final desta, o pesquisador terá reunido e analisado várias informações sobre o assunto pesquisado. A pesquisa descritiva proporciona uma nova visão sobre a realidade que já é conhecida. Para Campoy (2018, p. 56), "o investigador tem por finalidade descrever situações e eventos."

Consoante as afirmações dos autores, o problema de investigação descritiva nos permitiu com riqueza de detalhes, observar, analisar e descrever as características necessárias concernente ao tema em estudo, na busca de respostas para a natureza do problema desta pesquisa.

A investigação foi realizada na forma de pesquisa ação, segundo Elliot (1991, p. 69), a investigação ação é "um estudo de uma situação social com o objectivo de melhorar a qualidade da acção desenvolvida no seu interior". Neste sentido, nos permitiu realizar uma ação concernente ao desenvolvimento de um trabalho de modelagem matemática com o uso de tecnologias atrelado aos participantes dessa pesquisa.

Ainda, sendo classificada como de corte longitudinal considerando o exposto a seguir:

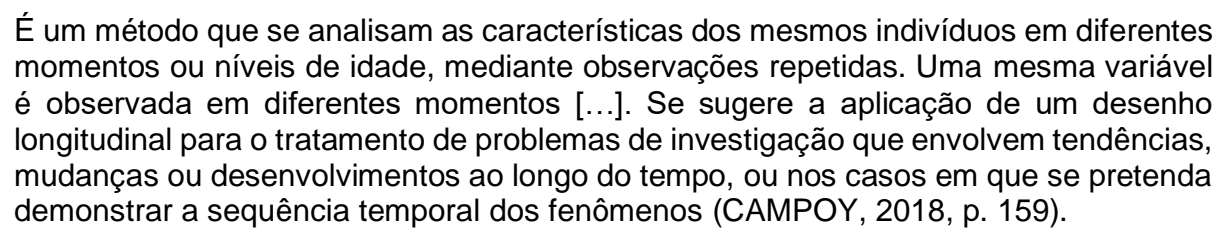

Trata-se de uma investigação de enfoque misto, a abordagem de pesquisa mista é uma visão emergente para a construção do conhecimento científico que considera os constructos significativos característicos da abordagem qualitativa, no entanto, também usa o rigor lógico matemática de técnicas estatísticas para construção de modelos que facilitem a tomada de decisão que convidam a transformar a realidade.

Instrumentos de coletas de dados: Questionário semiestruturado para alunos, entrevista para professores e observação participante.

Participantes: Uma turma do $3^{\circ}$ ano do ensino médio com 30 alunos e os professores, três, que 


\section{RECIMA21 - REVISTA CIENTÍFICA MULTIDISCIPLINAR ISSN 2675-6218}

A MODELAGEM MATEMÁTICA COMO PROPOSTA PEDAGÓGICANA PERSPECTIVA DO ENSINO DA MATEMÁTICA EM AMBIENTES INFORMATIZADOS NO ENSINO MÉDIO

ensinam matemática no ensino médio, da Escola de Referência em Ensino Médio Fábio da Silveira Barros.

Processo de seleção da amostra: É não probabilística e intencional. De acordo com Campoy, 2018, "los sujetos se seleccionan en relación a criterios del investigador", p.84.

\section{RESULTADOS}

\section{Objetivo Específico № 1:}

Assim, referente ao primeiro objetivo que é descrever as estratégias utilizadas pelos professores da Escola Fábio da Silveira Barros para ensinar matemática no ensino médio, podemos afirmar com base nos dados da pesquisa em profundidade que efetuamos, que os professores que lecionam matemática no ensino médio, dessa instituição de ensino, apropriam-se de uma metodologia construtiva, e em alguns casos, a metodologia tradicional, mas sempre enfatizam a busca por uma aproximação do conteúdo com a realidade do estudante.

Sobre os aportes para promoção do conhecimento, no caso as estratégias de ensino, que são técnicas utilizadas pelos professores com o objetivo de ajudar o aluno a construir seu conhecimento, foi possível apontar que os educadores fazem uso de aulas expositivas, explanação do conteúdo de forma clara e objetiva, fazem uso do livro didático do aluno, utilizam o quadro (lousa) assim como, o uso de materiais bibliográficos, tecnológicos (celular e computador), e materiais lúdicos, esses dois últimos recursos, citado por dois dos professores entrevistados, sendo um em cada caso.

\section{Objetivo Específico № 2:}

Assim, referente ao segundo objetivo de elaborar uma proposta pedagógica com desenvolvimento de atividades de Modelagem Matemática em ambientes informatizados, respaldada na Educação Matemática, essa foi elabora e desenvolvida a partir da temática escolhida pelos estudantes, que foi Obesidade, e com atividades desenvolvidas que ocorreram em três contextos: na sala de aula, no laboratório de informática improvisado e extras sala (farmácias e posto de saúde).

A proposta foi desenvolvida em 5 momentos. Em um primeiro momento, houve a escolha e delimitação do tema que poderia ser trabalhado.

No segundo momento houve a apresentação de vídeos sobre reportagens, percentuais de obesidade no país, hábitos alimentares, bem como discussão sobre essa temática com uma nutricionista ora convidada para essa ocasião.

Já no terceiro momento, ocorreu à atividade extra sala, onde os estudantes foram a farmácias e posto de saúde, verificarem seu peso e sua altura.

No quarto momento, houve a matematização e o modelo matemático, onde foram trabalhados conteúdos matemáticos associados ao uso de tecnologias.

No quinto e último momento, ocorreram às apresentações das equipes com seus respectivos resultados.

Objetivo Específico № 3: 


\section{RECIMA21 - REVISTA CIENTÍFICA MULTIDISCIPLINAR ISSN 2675-6218}

A MODELAGEM MATEMÁTICA COMO PROPOSTA PEDAGÓGICANA PERSPECTIVA DO ENSINO DA MATEMÁTICA EM AMBIENTES INFORMATIZADOS NO ENSINO MÉDIO
Rafaela Silva Bezerra do Nascimento

Para o terceiro objetivo que é descrever os resultados alcançados com a prática da nova metodologia apresentada, concluímos que o desenvolvimento das atividades com a proposta de modelagem matemática com tecnologia, possibilitou reconhecermos que para fazer um trabalho docente de qualidade é essencial que o professor saia da zona de conforto, interaja com os estudantes e esteja em constante formação cognitiva, social e, principalmente, humana.

Entendemos que diante dos resultados explicitados nos dados coletados dessa pesquisa, essa proposta metodológica terá papel fundamental na construção do conhecimento matemático desses estudantes, visto que ao utilizar uma proposta como essa, buscou-se levar ao estudante um ensino de qualidade, com significância e utilidade para o seu cotidiano.

$\mathrm{Na}$ última etapa que ocorrera a apresentação dos trabalhos desenvolvidos, percebeu-se a satisfação dos estudantes na concretização de todas as etapas que foram vivenciadas.

Com a pretensão de valorar e para reiterar nossas conclusões por meio do instrumento de observação, o questionário final constatou as contibruições do nosso trabalho, quando neste, evidenciam que o trabalho possibilitou aos alunos a conexão entre a matemática da escola com a matemática do seu cotidiano e com a conclusão do trabalho final, os resultados de uma aprendizagem significativa em relação ao conteúdo matemático e o uso de tecnologia. Nisso se confirma as palavras de Resende et. al. quando apontam que "através da Modelagem Matemática, os alunos despertem e desenvolvam a capacidade e o interesse de aprender a aprender, valorizando sempre o conhecimento vigente, possibilitando a correlação dos conteúdos já estudados com as necessidades do dia a dia" $(2015$, p. 2).

\section{CONCLUSÃO}

A pesquisa mostrou ser possível proporcionar experiências matemáticas significativas, úteis e estimulantes, envolvendo a escolha de um tema, investigação, criação de um modelo, ou mesmo matematização de uma situação, tudo isso associado ao uso de tecnologias. Além disso, mostrou como um trabalho diferenciado pode contribuir no processo de ensino e aprendizagem desta disciplina.

Levando em consideração os aspectos pesquisados, podemos relatar de uma forma geral que a turma onde ocorrera a pesquisa ação, da Erem Fábio da Silveira Barros, reflete a realidade de muitas outras escolas públicas de Ensino Médio. Estes estudantes apontavam para concepções negativas sobre a disciplina, a falta de uma matemática contextualizada, percebível em seu cotidiano, e depois da experiência com um trabalho de modelagem, com recursos tecnológicos, apresentaram mudanças positivas em relação a essas concepções.

No decorrer da aplicação dos instrumentos, percebemos que alguns pontos necessitam de aperfeiçoamentos para que o trabalho contemplando uma proposta de modelagem matemática e tecnologia, seja realmente algo efetivo e produza mudanças de qualidade, com resultados significativos no processo ensino aprendizagem.

De acordo com os dados coletados, os participantes da pesquisa expuseram suas opiniões, dificuldades, desejos e interesse na melhoria do ensino, nos aspectos referentes a uma proposta pedagógica diferenciada, para que esta, de fato possa contribuir para a construção do conhecimento 


\section{RECIMA21 - REVISTA CIENTÍFICA MULTIDISCIPLINAR ISSN 2675-6218}

A MODELAGEM MATEMÁTICA COMO PROPOSTA PEDAGÓGICANA PERSPECTIVA DO ENSINO DA MATEMÁTICA EM AMBIENTES INFORMATIZADOS NO ENSINO MÉDIO

matemático.

É conveniente ressaltar nesse momento que tanto o estudante apontou para a falta de uma matemática contextualizada, perceptível a seus olhos, como o professor apontou para a falta de conhecimentos necessários que os alunos devam possuir em determinados conteúdos, para que de fato possam dar continuidade ao seu trabalho, além disso, a escola não possuir meios para que possam ser acrescentados instrumentos tecnológicos às aulas, agregando valores e facilidades à aprendizagem.

Diante de todos os dados coletados e interpretados, concluiu-se que os estudantes, na maioria das vezes, não conseguem identificar uma relação dos conteúdos em uma situação real, ainda assim, ponderam sobre a necessidade de aprender uma matemática contextualizada.

Em relação aos professores, estes, procuram traçar um planejamento aonde venham atender as necessidades dos estudantes, mas nem sempre o colocam em prática, a falta de base dos estudantes requerida na disciplina, implicando assim no tempo disponível para a execução do mesmo.

De fato, as estratégias adotadas pelos professores apresentam fragilidades para alcançar os objetivos da aprendizagem, quando esses apontam para a falta de motivação e interesse por parte do estudante.

Ainda, a escola unidade de análise, não dispõe de recursos tecnológicos para serem utilizados pelos estudantes, como por exemplo um laboratório de informática. Assim, dispõe apenas de internet para o corpo docente da escola e, computador com Datashow para serem usados apenas na ministração das aulas e apresentação de trabalhos.

Efetivamente, esse contexto possui suas limitações, no entanto, ainda destacamos nessa conclusão, as contribuições alcançadas com a proposta da investigação, onde a mesma evidenciou mudanças nos sentimentos e visões dos alunos relacionados a uma aprendizagem significativa, comprovadas nos instrumentos utilizados na realização desse estudo.

Constatamos através do embasamento teórico, que a principal finalidade da Educação Matemática é garantir essa forma de pensamento e de leitura do mundo, pois a matemática está intimamente presente na sociedade tecnológica em que vivemos e pode ser encontrada sob várias formas no dia-a-dia. Com ela, o estudante aprende significativamente, quando consegue atribuir sentido e significado às ideias matemáticas, e sobre elas é capaz de pensar, estabelecer relações, justificar, analisar, discutir e criar. No entanto, existe uma distância do ideal para a realidade vivenciada. E que a inserção das tecnologias na escola para que possam ser utilizadas pelos estudantes, não depende unicamente dos professores, pois é um processo complexo que passa pela gestão e órgãos mantenedores, dependem de políticas públicas.

Tenhamos que, este trabalho, mostra apenas uma das possibilidades para se chegar a uma aprendizagem significativa com aportes tecnológicos, estejamos certo que:

Como sabemos, não existe uma receita para ensinarmos, bem como não há garantias de queesta ou aquela estratégia didática irá sempre funcionar. O que existe, de fato, é uma busca incessante, por parte de muitos professores, em procurar melhores caminhos para chegar ao objetivo almejado: a aprendizagem (ALBUQUERQUE, 2017, p. 124). 


\section{RECIMA21 - REVISTA CIENTÍFICA MULTIDISCIPLINAR ISSN 2675-6218}

A MODELAGEM MATEMÁTICA COMO PROPOSTA PEDAGÓGICANA PERSPECTIVA DO ENSINO DA MATEMÁTICA EM AMBIENTES INFORMATIZADOS NO ENSINO MEDIO Rafaela Silva Bezerra do Nascimento

\section{REFERÊNCIAS}

ALBUQUERQUE, E.; CARIO, S. A. F.; SUZIGAN, W. Em busca da inovação: interação universidadeempresa no Brasil. São Paulo: Autêntica, 2017.

ARAÚJO, J. L. Uma abordagem sócio-crítica da modelagem matemática: a perspectiva da educação matemática crítica. Alexandria, v. 2, n. 2, 2009.

BRASIL. Ministério da Educação. Secretaria de Educação Média e Tecnológica. Orientações Educacionais Complementares aos Parâmetros Curriculares Nacionais para o Ensino Médio. Brasilia: Ministério da Educação, 2011. Disponível em: http://cenp.edunet.sp.gov.br/Ens medio/em pcn.htm.

CAMPOY ARANDA, T. Metodologia de la investigación científica: manual para elaboración de tésis y trabajos de investigación. Asunción-Paraguay: Mardeben editora \& gráfica S. A., 2018.

CHAVES, M. I. de A. Repercussões de experiências com modelagem matemática em ações docentes. REMATEC, Natal-RN, ano 9, n. 17, 2014. Disponível em: http://www.rematec.net.br/index.php/inicio/issue/view/18/showToc.

D'AMBRÓSIO, U. Da realidade à ação reflexões sobre educação e matemática. Campinas: Editora da Unicamp, 1986.

ELLIOT, J. Action research for educational change. London: McGraw-Hill Education, 1991.

FRANCHI, R. H. de O. L. A modelagem matemática como estratégia de aprendizagem do Cálculo Diferencial e Integral nos cursos de Engenharia. 1993. Dissertação (Mestrado) - Instituto de Geociênciase Ciências Exatas, Universidade Estadual Paulista, Rio Claro, 1993.

FRANCHI, R. H. de O. L. Ambientes de Aprendizagem Fundamentados na Modelagem Matemática e na Informática como Possibilidades para a Educação Matemática. In: Modelagem Matemática Brasileira: pesquisas e práticas educacionais, Recife: Sbem, 2007. p. 177-193. v. 3.

Gil, A. C. Como elaborar projetos de pesquisa. 5. ed. São Paulo: Atlas, 2008.

JACOBINI, O. R. A Modelagem Matemática como instrumento de ação política na sala de aula. 2004. Tese (Doutorado) - Instituto de Geociências e Ciências Exatas, Universidade Estadual Paulista, Rio Claro, 2004.

MALHEIROS, A. P. A produção matemática dos alunos em um ambiente de modelagem. 2004. Dissertação (Mestrado em Educação Matemática) - Universidade Estadual Paulista, Rio Claro, 2004.

RESENDE, A. C.; FERREIRA, L. L.; BARBOSA, R. A. A modelagem matemática aplicada no acompanhamento do crescimento de um minhocário: um estudo de caso na disciplica de matemática no $2^{\circ}$ ano do Ensino Médio do curso técnico em Florestas do IFBAIANO - Campus Teixeira de Freitas. In.: XVI Encontro Baiano de Educação Matemática. Salvador, 2015. 